\section{ㄷ. REPORTS}

ISSN 1600-5368

\section{Crystal structure of bis[2-(1H-benzimid- azol-2-yl)-4-bromophenolato- $\left.\kappa^{2} N^{3}, O\right]-$ cobalt(II)}

\section{Yan Fan and Zhi-Rong Qu*}

Key Laboratory of Organosilicon Chemistry and Material Technology of the Ministry of Education, Hangzhou Normal University, Hangzhou 311121, People's Republic of China. *Correspondence e-mail: quzr@hznu.edu.cn

Received 25 August 2014; accepted 3 October 2014

Edited by D.-J. Xu, Zhejiang University (Yuquan Campus), China

The asymmetric unit of the title $\mathrm{Co}^{\mathrm{II}}$ complex, $\left[\mathrm{Co}\left(\mathrm{C}_{13} \mathrm{H}_{8} \mathrm{BrN}_{2} \mathrm{O}\right)_{2}\right]$, contains two independent molecules $(A$ and $B$ ). In both molecules, the $\mathrm{Co}^{\mathrm{II}}$ cation is $\mathrm{N}, \mathrm{O}$-chelated by two 2-(1H-benzimidazol-2-yl)-4-bromophenolate anions in a distorted tetrahedral geometry. In molecule $A$, both chelating rings display an envelope conformation, with the flap Co atom lying 0.614 (6) and 0.483 (6) $\AA$ from the mean planes of the remaining atoms. In molecule $B$, both chelating rings are approximately planar, the maximum deviations being 0.039 (4) and 0.076 (3) A. In the crystal, molecules are linked by classical $\mathrm{N}-\mathrm{H} \cdots \mathrm{O}$ hydrogen bonds and weak $\mathrm{C}-\mathrm{H} \cdots \mathrm{O}$ and $\mathrm{C}-\mathrm{H} \cdots \mathrm{Br}$ hydrogen bonds into a three-dimensional supramolecular network. Extensive $\pi-\pi$ stacking is observed between nearly parallel aromatic rings of adjacent molecules with centroid-centroid distances in the range 3.407 (3)3.850 (4) ^.

Keywords: crystal structure; cobalt(II); 2-(1H-benzimidazol-2-yl)-4bromophenolate anion; hydrogen bonds; $\pi-\pi$ stacking.

CCDC reference: 1027432

\section{Related literature}

For the crystal structures of related metal complexes with the ligand 2-(1H-benzoimidazol-2-yl)-4-bromophenolate, see: Li et al. (2002); Tong (2007).

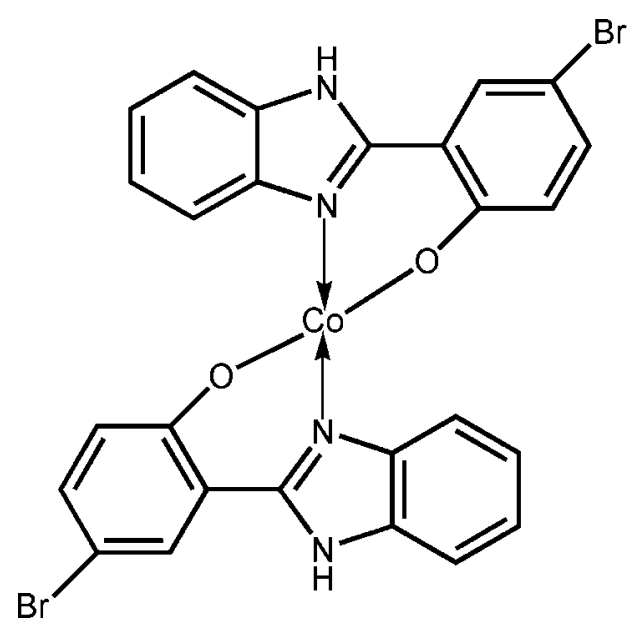

\section{Experimental}

2.1. Crystal data $\left[\mathrm{Co}\left(\mathrm{C}_{13} \mathrm{H}_{8} \mathrm{BrN}_{2} \mathrm{O}\right)_{2}\right]$ $M_{r}=635.16$

Monoclinic, $P 2_{1} / c$

$a=22.4334(16) \AA$

$b=8.3598(6) \AA$

$c=30.0748(16) \AA$

$\beta=125.323(4)^{\circ}$

$V=4601.9(5) \AA^{3}$

$Z=8$

Mo $K \alpha$ radiation

$\mu=4.25 \mathrm{~mm}^{-1}$

$T=293 \mathrm{~K}$

$0.30 \times 0.26 \times 0.20 \mathrm{~mm}$

\subsection{Data collection}

Bruker APEXII CCD diffractometer

Absorption correction: multi-scan (SADABS; Bruker, 2001)

$T_{\min }=0.258, T_{\max }=0.398$

2.3. Refinement

$R\left[F^{2}>2 \sigma\left(F^{2}\right)\right]=0.046$

$w R\left(F^{2}\right)=0.166$

$S=0.97$

10549 reflections

631 parameters

$\mathrm{H}$-atom parameters constrained

$\Delta \rho_{\max }=0.71{\mathrm{e} \AA^{-3}}^{-3}$

$\Delta \rho_{\min }=-0.65$ e $\AA^{-3}$
29475 measured reflections 10549 independent reflections $R_{\text {int }}=0.053$ 5904 reflections with $I>2 \sigma(I)$

Table 1

Selected bond lengths $(\AA)$.

\begin{tabular}{llll}
\hline $\mathrm{Co} 1-\mathrm{O} 2$ & $1.912(4)$ & $\mathrm{Co} 2-\mathrm{O} 1$ & $1.930(4)$ \\
$\mathrm{Co} 1-\mathrm{O} 3$ & $1.903(4)$ & $\mathrm{Co} 2-\mathrm{O} 4$ & $1.912(4)$ \\
$\mathrm{Co} 1-\mathrm{N} 2$ & $1.975(4)$ & $\mathrm{Co} 2-\mathrm{N} 6$ & $1.961(4)$ \\
$\mathrm{Co} 1-\mathrm{N} 3$ & $1.967(4)$ & $\mathrm{Co} 2-\mathrm{N} 7$ & $1.957(4)$ \\
\hline
\end{tabular}


Table 2

Hydrogen-bond geometry $\left(\AA{ }^{\circ}\right)$.

\begin{tabular}{lcclc}
\hline$D-\mathrm{H} \cdots A$ & $D-\mathrm{H}$ & $\mathrm{H} \cdots A$ & $D \cdots A$ & $D-\mathrm{H} \cdots A$ \\
\hline $\mathrm{N} 1-\mathrm{H} 1 A \cdots \mathrm{O} 1^{\mathrm{i}}$ & 0.86 & 2.19 & $2.895(5)$ & 139 \\
$\mathrm{~N} 4-\mathrm{H} 4 A \cdots \mathrm{O} 2^{\mathrm{ii}}$ & 0.86 & 2.09 & $2.849(5)$ & 147 \\
$\mathrm{~N} 5-\mathrm{H} 5 A \cdots \mathrm{O} 4^{\text {iii }}$ & 0.86 & 2.41 & $3.066(5)$ & 133 \\
$\mathrm{~N} 8-\mathrm{H} 8 A \cdots \mathrm{O} 3^{\text {iv }}$ & 0.86 & 2.17 & $2.808(5)$ & 131 \\
$\mathrm{C} 4-\mathrm{H} 4 \cdots \mathrm{Br}^{\mathrm{i}}$ & 0.93 & 2.88 & $3.703(6)$ & 149 \\
$\mathrm{C} 6-\mathrm{H} 6 \cdots \mathrm{Br}^{\mathrm{v}}$ & 0.93 & 2.91 & $3.685(6)$ & 142 \\
$\mathrm{C} 35-\mathrm{H} 35 \cdots \mathrm{O} 2^{\mathrm{ii}}$ & 0.93 & 2.54 & $3.394(7)$ & 153 \\
\hline Symmetry codes: & (i) $-x+1, y-\frac{1}{2},-z+\frac{3}{2} ; \quad$ (ii) $-x+2,-y+1,-z+2 ; \quad$ (iii) \\
$-x+1,-y+2,-z+2 ;($ iv $)-x+1, y+\frac{1}{2},-z+\frac{3}{2} ;$ (v) $x,-y+\frac{3}{2}, z+\frac{1}{2}$. &
\end{tabular}

Data collection: APEX2 (Bruker, 2007); cell refinement: SAINT (Bruker, 2007); data reduction: $S A I N T$; $\operatorname{program}(\mathrm{s})$ used to solve structure: SHELXTL (Sheldrick, 2008); program(s) used to refine structure: SHELXTL; molecular graphics: SHELXTL; software used to prepare material for publication: SHELXTL (Sheldrick, 2008).

\section{Acknowledgements}

This work was supported by a start-up grant from Hangzhou Normal University, China.

Supporting information for this paper is available from the IUCr electronic archives (Reference: XU5818).

\section{References}

Bruker (2001). SADABS. Bruker AXS Inc., Madison, Wisconsin, USA.

Bruker (2007). APEX2 and SAINT. Bruker AXS Inc., Madison, Wisconsin, USA.

Li, J., Zhang, F.-X. \& Shi, Q.-Z. (2002). Chin. J. Inorg. Chem. 18, 643-646. Sheldrick, G. M. (2008). Acta Cryst. A64, 112-122.

Tong, Y.-P. (2007). Chin. J. Struct. Chem. 26, 143-145. 


\section{supporting information}

Acta Cryst. (2014). E70, m363-m364 [doi:10.1107/S1600536814021813]

\section{Crystal structure of bis[2-(1 H-benzimidazol-2-yl)-4-bromophenolato- $\left.\kappa^{2} N^{3}, O\right]$ cobalt(II)}

\section{Yan Fan and Zhi-Rong Qu}

\section{S1. Comment}

The asymmetric unit of the title compound contains two crystallographically independent molecules and bond lengths and angles are in the normal range (Li et al., 2002; Tong, 2007). The Co(II) atom is coordinated by two $\mathrm{N}$ atoms and two $\mathrm{O}$ atoms to give a distorted tetrahedral geometry (Fig. 1). The benzimidazole and bromophenyl groups are nearly coplanar; the dihedral angle between them is $4.2(3)^{\circ}$. The angles between the two ligand planes in each independent molecule are $71.66(5)^{\circ}$ and $63.66(5)^{\circ}$, respectively. The crystal structure is stabilized by $\mathrm{N}-\mathrm{H} \cdots \mathrm{O}$ hydrogen bonds linking molecules into a three-dimensional network structure (Fig. 2). The structure is further stabilized by $\pi-\pi$ stacking interactions, the centroid-to-centroid separations are ranged from 3.407 (3) to 3.850 (4) $\AA$.

\section{S2. Experimental}

Synthesis of the ligand: The ligands were prepared by the reaction of the addition products of 2-hydroxy-5-bromobenzaldehyde (6 mmol $1.2 \mathrm{~g})$ and $\mathrm{NaHSO}_{3}(6 \mathrm{mmol} 0.65 \mathrm{~g})$ were stirred at room temperature in ethanol $(25 \mathrm{ml})$ and a precipitate is formed after $4 \mathrm{~h}$ reaction, then, $o$-phenylenediamine $(6 \mathrm{mmol}, 0.65 \mathrm{~g})$ and $25 \mathrm{ml} \mathrm{DMF}$ were added to this mixture. After $2 \mathrm{~h}$ reflux the solution was poured into 10-times water. The benzimidazole compound was filtered, dried and crystallized from ethanol.

Synthesis of the complex [Co( $\left.\left.\mathrm{C}_{26} \mathrm{H}_{16} \mathrm{~N}_{4} \mathrm{O}_{2} \mathrm{Br}_{2}\right)\right]_{2}:$ 2-(1H-benzimidazol-2-yl)-4-bromophenol (0.2 mmol, $\left.58 \mathrm{mg}\right)$ was dissolved in DMF $(6 \mathrm{ml})$ and $\mathrm{CoCl}_{2} \cdot 6 \mathrm{H}_{2} \mathrm{O}(0.1 \mathrm{mmol} 24 \mathrm{mg})$ was dissolved in $\mathrm{H}_{2} \mathrm{O}(6 \mathrm{ml})$, and the mixture poured into a $25 \mathrm{ml}$ dicting kettle, then maintaining $393 \mathrm{~K}$ for $3 \mathrm{~d}$, allowed to reach room temperate, and red block-shaped crystals suitable for X-ray diffraction were obtained.

\section{S3. Refinement}

$\mathrm{H}$ atoms were placed in calculated positions with $\mathrm{C}-\mathrm{H}=0.97-0.93 \AA$ and $\mathrm{N}-\mathrm{H}=0.86 \AA$, and refined in riding mode, $\mathrm{U}_{\mathrm{iso}}(\mathrm{H})=1.2 \mathrm{U}_{\mathrm{eq}}(\mathrm{C}, \mathrm{N})$. 


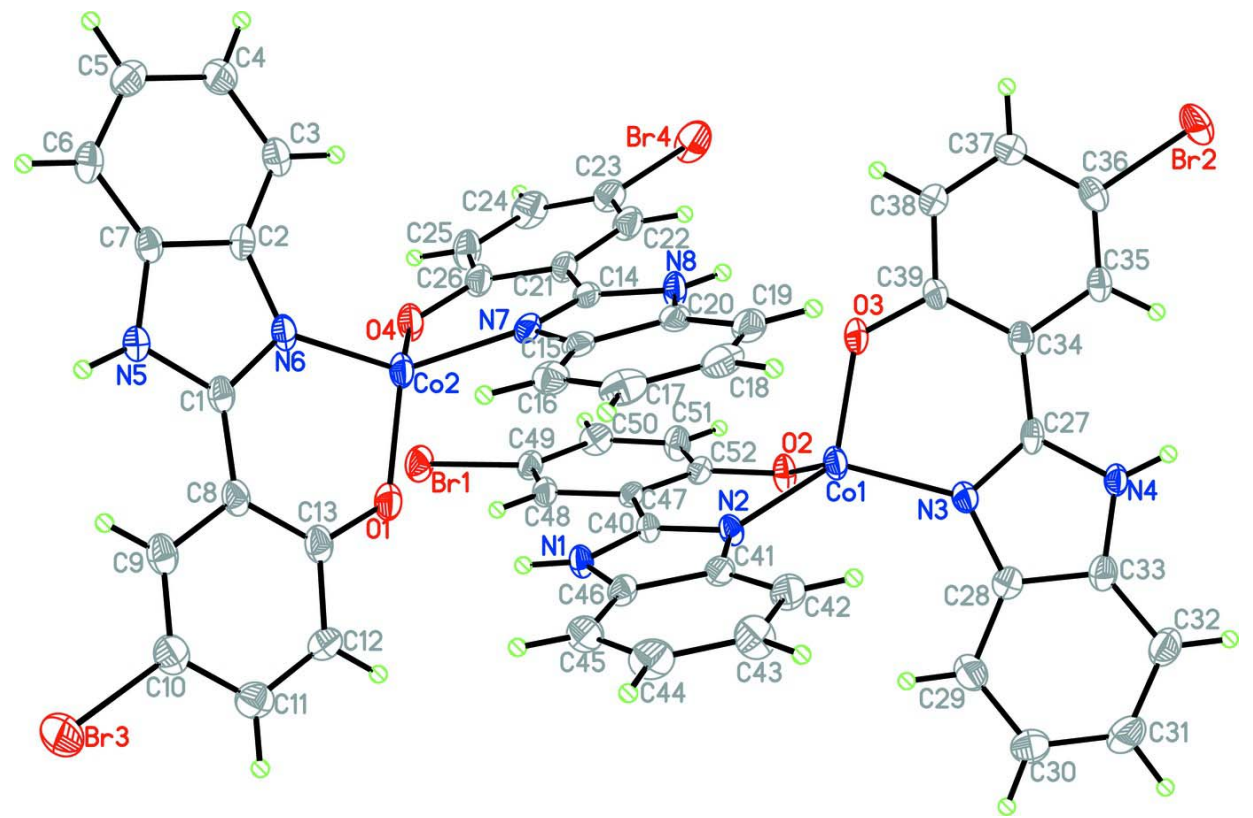

\section{Figure 1}

A view of the asymmetric unit of the title compound with atomic numbering scheme. Displacement ellipsoids were drawn at the $30 \%$ probability level and all $\mathrm{H}$ atoms have been omitted for clarity.

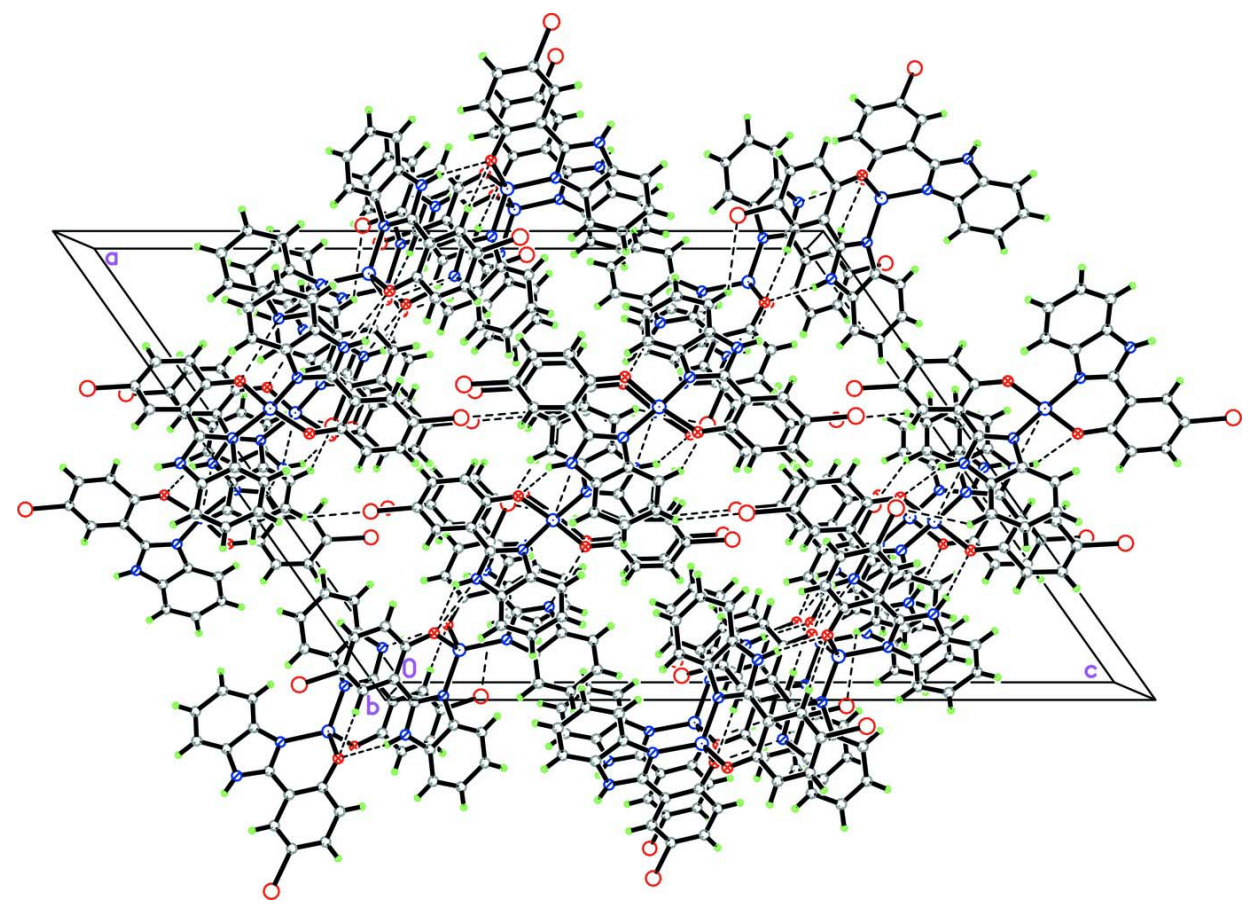

Figure 2

Packing diagram of the title compound viewed along the $c$ axis. Hydrogen bonds are shown as dashed lines. 
Bis[2-(1H-benzimidazol-2-yl)-4-bromophenolato- $\left.\kappa^{2} N^{3}, O\right]$ cobalt(II)

Crystal data

$\left[\mathrm{Co}\left(\mathrm{C}_{13} \mathrm{H}_{8} \mathrm{BrN}_{2} \mathrm{O}\right)_{2}\right]$

$M_{r}=635.16$

Monoclinic, $P 2{ }_{1} / c$

$a=22.4334(16) \AA$

$b=8.3598(6) \AA$

$c=30.0748(16) \AA$

$\beta=125.323(4)^{\circ}$

$V=4601.9(5) \AA^{3}$

$Z=8$

Data collection

Bruker APEXII CCD

diffractometer

Radiation source: fine-focus sealed tube

Graphite monochromator

$\varphi$ and $\omega$ scans

Absorption correction: multi-scan

(SADABS; Bruker, 2001)

$T_{\min }=0.258, T_{\max }=0.398$

Refinement

Refinement on $F^{2}$

Least-squares matrix: full

$R\left[F^{2}>2 \sigma\left(F^{2}\right)\right]=0.046$

$w R\left(F^{2}\right)=0.166$

$S=0.97$

10549 reflections

631 parameters

0 restraints

Primary atom site location: structure-invariant

direct methods
$F(000)=2503$

$D_{\mathrm{x}}=1.834 \mathrm{Mg} \mathrm{m}^{-3}$

Mo $K \alpha$ radiation, $\lambda=0.71073 \AA$

Cell parameters from 29475 reflections

$\theta=1.1-27.5^{\circ}$

$\mu=4.25 \mathrm{~mm}^{-1}$

$T=293 \mathrm{~K}$

Block, red

$0.30 \times 0.26 \times 0.20 \mathrm{~mm}$

29475 measured reflections

10549 independent reflections

5904 reflections with $I>2 \sigma(I)$

$R_{\text {int }}=0.053$

$\theta_{\max }=27.5^{\circ}, \theta_{\min }=1.1^{\circ}$

$h=-29 \rightarrow 22$

$k=-10 \rightarrow 10$

$l=-36 \rightarrow 39$

Secondary atom site location: difference Fourier map

Hydrogen site location: inferred from neighbouring sites

$\mathrm{H}$-atom parameters constrained

$w=1 /\left[\sigma^{2}\left(F_{0}^{2}\right)+(0.0908 P)^{2}\right]$

where $P=\left(F_{\mathrm{o}}^{2}+2 F_{\mathrm{c}}^{2}\right) / 3$

$(\Delta / \sigma)_{\max }=0.001$

$\Delta \rho_{\max }=0.71$ e $\AA^{-3}$

$\Delta \rho_{\min }=-0.65$ e $\AA^{-3}$

Special details

Geometry. All e.s.d.'s (except the e.s.d. in the dihedral angle between two 1.s. planes) are estimated using the full covariance matrix. The cell e.s.d.'s are taken into account individually in the estimation of e.s.d.'s in distances, angles and torsion angles; correlations between e.s.d.'s in cell parameters are only used when they are defined by crystal symmetry. An approximate (isotropic) treatment of cell e.s.d.'s is used for estimating e.s.d.'s involving 1.s. planes.

Refinement. Refinement of $F^{2}$ against ALL reflections. The weighted $R$-factor $w R$ and goodness of fit $S$ are based on $F^{2}$, conventional $R$-factors $R$ are based on $F$, with $F$ set to zero for negative $F^{2}$. The threshold expression of $F^{2}>\sigma\left(F^{2}\right)$ is used only for calculating $R$-factors(gt) etc. and is not relevant to the choice of reflections for refinement. $R$-factors based on $F^{2}$ are statistically about twice as large as those based on $F$, and $R$ - factors based on ALL data will be even larger.

Fractional atomic coordinates and isotropic or equivalent isotropic displacement parameters $\left(\AA^{2}\right)$

\begin{tabular}{lllll}
\hline & $x$ & $y$ & $z$ & $U_{\text {iso }} * / U_{\text {eq }}$ \\
\hline Br1 & $0.58763(3)$ & $0.94519(8)$ & $0.67243(2)$ & $0.04708(19)$ \\
Br2 & $0.96705(4)$ & $-0.01562(9)$ & $1.09681(2)$ & $0.0546(2)$ \\
Co1 & $0.90832(4)$ & $0.46833(10)$ & $0.87113(3)$ & $0.0345(2)$ \\
O2 & $0.85783(19)$ & $0.6568(5)$ & $0.86818(12)$ & $0.0383(10)$ \\
O3 & $0.86633(19)$ & $0.2923(5)$ & $0.88397(12)$ & $0.0439(10)$
\end{tabular}




\begin{tabular}{|c|c|c|c|c|}
\hline N2 & $0.8868(2)$ & $0.5101(5)$ & $0.79854(15)$ & $0.0278(10)$ \\
\hline $\mathrm{N} 1$ & $0.8152(2)$ & $0.5734(5)$ & $0.71102(15)$ & $0.0298(10)$ \\
\hline $\mathrm{H} 1 \mathrm{~A}$ & 0.7795 & 0.6141 & 0.6809 & $0.036^{*}$ \\
\hline N3 & $1.0079(2)$ & $0.4220(5)$ & $0.93502(15)$ & $0.0321(10)$ \\
\hline N4 & 1.0909 (2) & $0.3509(5)$ & $1.02030(15)$ & $0.0312(10)$ \\
\hline $\mathrm{H} 4 \mathrm{~A}$ & 1.1100 & 0.3093 & 1.0520 & $0.037^{*}$ \\
\hline $\mathrm{C} 41$ & $0.9158(3)$ & $0.4410(7)$ & $0.77260(19)$ & $0.0354(13)$ \\
\hline $\mathrm{C} 28$ & $1.0753(3)$ & $0.4820(7)$ & $0.9510(2)$ & $0.0337(13)$ \\
\hline $\mathrm{C} 36$ & $0.9396(3)$ & $0.0799(7)$ & $1.0302(2)$ & $0.0380(14)$ \\
\hline $\mathrm{C} 40$ & $0.8271(3)$ & $0.5913(6)$ & $0.76042(17)$ & $0.0260(11)$ \\
\hline $\mathrm{C} 27$ & $1.0192(3)$ & $0.3386(7)$ & $0.97719(17)$ & $0.0298(12)$ \\
\hline $\mathrm{C} 46$ & $0.8705(3)$ & $0.4784(7)$ & $0.7173(2)$ & $0.0301(12)$ \\
\hline $\mathrm{C} 45$ & $0.8855(3)$ & $0.4264(8)$ & $0.6813(2)$ & $0.0437(15)$ \\
\hline $\mathrm{H} 45$ & 0.8550 & 0.4512 & 0.6444 & $0.052 *$ \\
\hline $\mathrm{C} 48$ & $0.7168(3)$ & $0.7559(7)$ & $0.72527(18)$ & $0.0340(13)$ \\
\hline H48 & 0.7050 & 0.7419 & 0.6904 & $0.041 *$ \\
\hline $\mathrm{C} 33$ & $1.1276(3)$ & $0.4405(7)$ & $1.0050(2)$ & $0.0365(13)$ \\
\hline $\mathrm{C} 49$ & $0.6728(3)$ & $0.8449(7)$ & 0.73248 (19) & $0.0335(13)$ \\
\hline $\mathrm{C} 35$ & $0.9864(3)$ & $0.1691(7)$ & $1.02718(18)$ & $0.0315(12)$ \\
\hline H35 & 1.0341 & 0.1807 & 1.0580 & $0.038 *$ \\
\hline $\mathrm{C} 34$ & $0.9655(3)$ & $0.2465(7)$ & $0.97832(17)$ & $0.0303(12)$ \\
\hline $\mathrm{C} 43$ & 0.9927 (3) & $0.2955(8)$ & $0.7576(2)$ & $0.0488(16)$ \\
\hline H43 & 1.0336 & 0.2323 & 0.7702 & $0.059^{*}$ \\
\hline $\mathrm{C} 44$ & $0.9470(3)$ & $0.3370(9)$ & $0.7022(2)$ & $0.0534(18)$ \\
\hline $\mathrm{H} 44$ & 0.9591 & 0.3023 & 0.6790 & $0.064 *$ \\
\hline $\mathrm{C} 31$ & $1.2178(3)$ & $0.5794(9)$ & $1.0040(3)$ & $0.0572(18)$ \\
\hline H31 & 1.2655 & 0.6161 & 1.0215 & $0.069^{*}$ \\
\hline C50 & $0.6885(3)$ & $0.8666(7)$ & $0.7841(2)$ & $0.0408(14)$ \\
\hline $\mathrm{H} 50$ & 0.6569 & 0.9242 & 0.7886 & $0.049^{*}$ \\
\hline $\mathrm{C} 47$ & $0.7801(2)$ & $0.6842(6)$ & $0.76986(17)$ & $0.0254(11)$ \\
\hline $\mathrm{C} 42$ & $0.9773(3)$ & $0.3479(8)$ & $0.7936(2)$ & $0.0468(16)$ \\
\hline H42 & 1.0071 & 0.3213 & 0.8304 & $0.056^{*}$ \\
\hline C37 & $0.8683(3)$ & $0.0559(7)$ & $0.9848(2)$ & $0.0454(15)$ \\
\hline H37 & 0.8360 & -0.0078 & 0.9869 & $0.054^{*}$ \\
\hline $\mathrm{C} 51$ & $0.7506(3)$ & $0.8027(7)$ & $0.82781(19)$ & $0.0388(14)$ \\
\hline H51 & 0.7617 & 0.8204 & 0.8624 & $0.047^{*}$ \\
\hline C32 & $1.2000(3)$ & $0.4847(8)$ & $1.0325(3)$ & $0.0505(17)$ \\
\hline H32 & 1.2349 & 0.4526 & 1.0682 & $0.061^{*}$ \\
\hline C39 & $0.8932(3)$ & $0.2255(7)$ & $0.93201(18)$ & $0.0326(13)$ \\
\hline C52 & $0.7979(3)$ & $0.7121(6)$ & $0.82274(18)$ & $0.0292(12)$ \\
\hline $\mathrm{C} 29$ & $1.0957(3)$ & $0.5776(8)$ & $0.9235(2)$ & $0.0474(16)$ \\
\hline $\mathrm{H} 29$ & 1.0613 & 0.6101 & 0.8877 & $0.057^{*}$ \\
\hline $\mathrm{C} 30$ & $1.1663(3)$ & $0.6213(10)$ & $0.9498(3)$ & $0.063(2)$ \\
\hline H30 & 1.1808 & 0.6805 & 0.9314 & $0.076^{*}$ \\
\hline $\mathrm{C} 38$ & $0.8472(3)$ & $0.1294(8)$ & $0.9369(2)$ & $0.0451(15)$ \\
\hline H38 & 0.7997 & 0.1137 & 0.9063 & $0.054 *$ \\
\hline $\mathrm{Br} 3$ & $0.33496(4)$ & $1.05417(12)$ & $1.10266(3)$ & $0.0769(3)$ \\
\hline $\mathrm{Br} 4$ & $0.39831(4)$ & $0.96643(12)$ & $0.64170(3)$ & $0.0711(3)$ \\
\hline
\end{tabular}




\begin{tabular}{|c|c|c|c|c|}
\hline $\mathrm{Co} 2$ & $0.37925(4)$ & $0.92802(10)$ & $0.87904(3)$ & $0.0363(2)$ \\
\hline $\mathrm{O} 1$ & $0.3195(2)$ & $1.0374(5)$ & $0.89651(14)$ & $0.0451(11)$ \\
\hline O4 & $0.4319(2)$ & $1.0277(5)$ & $0.85430(14)$ & $0.0430(10)$ \\
\hline N6 & $0.4425(2)$ & $0.8299(6)$ & $0.95131(15)$ & $0.0340(11)$ \\
\hline N5 & $0.4949(2)$ & $0.7840(5)$ & $1.03884(15)$ & $0.0323(10)$ \\
\hline H5A & 0.5032 & 0.7876 & 1.0705 & $0.039 *$ \\
\hline N7 & $0.3160(2)$ & $0.8034(6)$ & $0.81237(15)$ & $0.0339(11)$ \\
\hline N8 & $0.2534(2)$ & $0.7465(6)$ & $0.72462(15)$ & $0.0356(11)$ \\
\hline H8A & 0.2400 & 0.7475 & 0.6914 & $0.043 *$ \\
\hline $\mathrm{C} 13$ & $0.3259(3)$ & $1.0325(7)$ & $0.9430(2)$ & $0.0382(14)$ \\
\hline $\mathrm{C} 21$ & $0.3638(3)$ & $0.9139(7)$ & $0.76286(19)$ & $0.0359(13)$ \\
\hline $\mathrm{C} 14$ & $0.3128(3)$ & $0.8257(7)$ & $0.76724(18)$ & $0.0324(13)$ \\
\hline $\mathrm{C} 8$ & 0.3808 (3) & 0.9497 (7) & $0.9903(2)$ & $0.0355(13)$ \\
\hline C3 & $0.5332(3)$ & $0.6743(7)$ & $0.9445(2)$ & $0.0425(15)$ \\
\hline H3 & 0.5116 & 0.6953 & 0.9078 & $0.051^{*}$ \\
\hline C19 & $0.1575(3)$ & $0.5672(7)$ & $0.7178(2)$ & $0.0435(15)$ \\
\hline H19 & 0.1312 & 0.5432 & 0.6809 & $0.052 *$ \\
\hline C6 & $0.5994(3)$ & $0.6084(7)$ & $1.0561(2)$ & $0.0409(15)$ \\
\hline H6 & 0.6213 & 0.5861 & 1.0928 & $0.049^{*}$ \\
\hline $\mathrm{C} 2$ & $0.5037(3)$ & $0.7354(7)$ & $0.97079(18)$ & $0.0314(12)$ \\
\hline C9 & 0.3807 (3) & $0.9555(7)$ & $1.0370(2)$ & $0.0393(14)$ \\
\hline H9 & 0.4166 & 0.9005 & 1.0682 & $0.047 *$ \\
\hline $\mathrm{C} 4$ & $0.5948(3)$ & $0.5823(8)$ & $0.9741(2)$ & $0.0478(16)$ \\
\hline $\mathrm{H} 4$ & 0.6156 & 0.5406 & 0.9573 & $0.057^{*}$ \\
\hline $\mathrm{C} 15$ & $0.2582(3)$ & $0.7042(7)$ & 0.79894 (19) & $0.0344(13)$ \\
\hline $\mathrm{C} 26$ & $0.4212(3)$ & $1.0058(8)$ & $0.8068(2)$ & $0.0404(15)$ \\
\hline $\mathrm{C} 1$ & $0.4374(3)$ & $0.8591(7)$ & $0.99257(18)$ & $0.0319(12)$ \\
\hline $\mathrm{C} 10$ & $0.3293(3)$ & $1.0395(8)$ & $1.0377(2)$ & $0.0455(16)$ \\
\hline $\mathrm{C} 17$ & $0.1765(3)$ & $0.5397(7)$ & $0.8052(3)$ & $0.0471(16)$ \\
\hline H17 & 0.1611 & 0.4947 & 0.8253 & $0.057^{*}$ \\
\hline $\mathrm{C} 20$ & $0.2184(3)$ & $0.6648(6)$ & $0.7434(2)$ & $0.0303(12)$ \\
\hline $\mathrm{C} 24$ & $0.4628(3)$ & $1.0748(9)$ & $0.7502(3)$ & $0.0521(17)$ \\
\hline $\mathrm{H} 24$ & 0.4951 & 1.1293 & 0.7457 & $0.062 *$ \\
\hline $\mathrm{C} 5$ & $0.6266(3)$ & $0.5506(8)$ & $1.0284(2)$ & $0.0478(16)$ \\
\hline H5 & 0.6684 & 0.4873 & 1.0471 & $0.057^{*}$ \\
\hline $\mathrm{C} 7$ & $0.5373(3)$ & $0.7018(7)$ & $1.02606(18)$ & $0.0307(12)$ \\
\hline $\mathrm{C} 25$ & 0.4695 (3) & $1.0851(8)$ & $0.7981(2)$ & $0.0454(16)$ \\
\hline $\mathrm{H} 25$ & 0.5072 & 1.1465 & 0.8261 & $0.054^{*}$ \\
\hline C16 & $0.2367(3)$ & $0.6371(7)$ & $0.8302(2)$ & $0.0381(13)$ \\
\hline H16 & 0.2628 & 0.6582 & 0.8673 & $0.046^{*}$ \\
\hline $\mathrm{C} 22$ & $0.3593(3)$ & $0.9059(9)$ & $0.7142(2)$ & $0.0464(16)$ \\
\hline $\mathrm{H} 22$ & 0.3219 & 0.8463 & 0.6853 & $0.056^{*}$ \\
\hline $\mathrm{C} 12$ & $0.2744(3)$ & $1.1173(8)$ & $0.9458(2)$ & $0.0425(14)$ \\
\hline H12 & 0.2374 & 1.1715 & 0.9150 & $0.051 *$ \\
\hline $\mathrm{C} 23$ & $0.4072(3)$ & $0.9819(8)$ & $0.7081(2)$ & $0.0457(16)$ \\
\hline C11 & $0.2759(3)$ & $1.1239(8)$ & $0.9918(2)$ & $0.0445(15)$ \\
\hline H11 & 0.2416 & 1.1844 & 0.9925 & $0.053^{*}$ \\
\hline C18 & $0.1387(3)$ & $0.5089(8)$ & $0.7500(3)$ & $0.0480(16)$ \\
\hline
\end{tabular}


supporting information

\begin{tabular}{|c|c|c|c|}
\hline $\mathrm{H} 18$ & 0.0976 & 0.4437 & 0.7340 \\
\hline
\end{tabular}

Atomic displacement parameters $\left(\AA^{2}\right)$

\begin{tabular}{|c|c|c|c|c|c|c|}
\hline & $U^{11}$ & $U^{22}$ & $U^{33}$ & $U^{12}$ & $U^{13}$ & $U^{23}$ \\
\hline Br1 & 0.0315 & $0.0561(5)$ & 0.0418 & $0.0132(3)$ & $0.0143(3)$ & $0.0101(3)$ \\
\hline $\mathrm{Br} 2$ & $0.0679(5)$ & $0.0629(5)$ & 0.0378 & $0.0107(4)$ & $0.0334(3)$ & $0.0182(3)$ \\
\hline Col & $0.0303(4)$ & $0.0474(5)$ & 0.0209 & 0.0058 & 0.0121 & $0.0074(3)$ \\
\hline $\mathrm{O} 2$ & $0.036(2)$ & $0.052(3)$ & $0.0194(16)$ & 0.0137 (19) & $0.0118(15)$ & $0.0036(16)$ \\
\hline $\mathrm{O} 3$ & $0.034(2)$ & $0.063(3)$ & $0.0200(16)$ & $-0.001(2)$ & $0.0066(15)$ & $0.0088(17)$ \\
\hline N2 & $0.027(2)$ & $0.031(3)$ & 0.0247 (19) & $0.010(2)$ & $0.0150(18)$ & $0.0085(18)$ \\
\hline N1 & $0.025(2)$ & 0.039 & $0.0226(19)$ & $0.010(2)$ & $0.0126(17)$ & $0.0071(18)$ \\
\hline N3 & $0.032(2)$ & $0.038(3)$ & $0.0239(19)$ & $0.002(2)$ & $0.0144(18)$ & 0.0046 (19) \\
\hline N4 & $0.026(2)$ & $0.039(3)$ & 0.0194 (18) & $0.002(2)$ & 0.0085 (18) & $0.0029(18)$ \\
\hline C41 & $0.029(3)$ & $0.055(4)$ & $0.026(2)$ & $-0.006(3)$ & $0.017(2)$ & $-0.002(2)$ \\
\hline $\mathrm{C} 28$ & $0.033(3)$ & $0.036(3)$ & $0.033(3)$ & $0.002(3)$ & $0.019(2)$ & $-0.002(2)$ \\
\hline $\mathrm{C} 36$ & $0.047(3)$ & $0.042(4)$ & $0.027(2)$ & $0.005(3)$ & $0.023(3)$ & $0.006(2)$ \\
\hline $\mathrm{C} 40$ & $0.024(3)$ & $0.027(3)$ & $0.022(2)$ & $0.005(2)$ & $0.010(2)$ & $0.004(2)$ \\
\hline $\mathrm{C} 27$ & 0.029 (3) & $0.035(3)$ & $0.017(2)$ & $0.008(2)$ & 0.009 (2) & $-0.002(2)$ \\
\hline $\mathrm{C} 46$ & $0.023(3)$ & $0.031(3)$ & $0.034(3)$ & $0.005(2)$ & $0.015(2)$ & $0.002(2)$ \\
\hline $\mathrm{C} 45$ & $0.045(3)$ & $0.061(5)$ & $0.035(3)$ & $-0.005(3)$ & $0.029(3)$ & -0.007 \\
\hline $\mathrm{C} 48$ & $0.028(3)$ & $0.049(4)$ & $0.022(2)$ & $-0.002(3)$ & $0.012(2)$ & $0.001(2)$ \\
\hline C33 & $0.031(3)$ & $0.042(4)$ & $0.032(3)$ & $0.009(3)$ & $0.016(2)$ & $0.003(2)$ \\
\hline C49 & $0.024(3)$ & $0.043(4)$ & $0.030(2)$ & $0.003(3)$ & $0.013(2)$ & $0.002(2)$ \\
\hline $\mathrm{C} 35$ & $0.030(3)$ & $0.041(4)$ & $0.021(2)$ & $0.010(3)$ & $0.013(2)$ & $0.002(2)$ \\
\hline $\mathrm{C} 34$ & $0.030(3)$ & $0.037(3)$ & $0.021(2)$ & $0.004(2)$ & $0.013(2)$ & $0.000(2)$ \\
\hline $\mathrm{C} 43$ & $0.047(4)$ & $0.050(4)$ & $0.060(4)$ & $0.023(3)$ & $0.036(3)$ & $0.011(3)$ \\
\hline $\mathrm{C} 44$ & $0.053(4)$ & $0.072(5)$ & $0.055(4)$ & $-0.002(4)$ & $0.043(3)$ & -0.010 \\
\hline C31 & $0.034(3)$ & $0.068(5)$ & $0.067(4)$ & 0.003 & $0.028(3)$ & $0.003(4)$ \\
\hline $\mathrm{C} 50$ & 0.042 & $0.043(4)$ & $0.044(3)$ & $0.001(3)$ & $0.029(3)$ & $-0.007(3)$ \\
\hline $\mathrm{C} 47$ & $0.022(2)$ & $0.029(3)$ & $0.024(2)$ & $-0.003(2)$ & $0.013(2)$ & $-0.003(2)$ \\
\hline $\mathrm{C} 42$ & $0.033(3)$ & $0.071(5)$ & $0.043(3)$ & $0.006(3)$ & $0.026(3)$ & $0.008(3)$ \\
\hline $\mathrm{C} 37$ & $0.041(3)$ & $0.049(4)$ & $0.041(3)$ & $-0.006(3)$ & $0.021(3)$ & $0.007(3)$ \\
\hline C51 & $0.038(3)$ & $0.055(4)$ & $0.024(2)$ & $0.005(3)$ & $0.018(2)$ & $-0.004(2)$ \\
\hline $\mathrm{C} 32$ & $0.031(3)$ & $0.069(5)$ & $0.045(3)$ & $0.005(3)$ & $0.018(3)$ & $0.004(3)$ \\
\hline C39 & $0.033(3)$ & $0.039(4)$ & $0.022(2)$ & $0.002(3)$ & $0.013(2)$ & $0.005(2)$ \\
\hline $\mathrm{C} 52$ & $0.024(3)$ & $0.031(3)$ & $0.026(2)$ & $-0.001(2)$ & $0.011(2)$ & $-0.002(2)$ \\
\hline $\mathrm{C} 29$ & $0.049(4)$ & $0.048(4)$ & 0.045 & $0.006(3)$ & $0.027(3)$ & $0.012(3)$ \\
\hline $\mathrm{C} 30$ & $0.041(4)$ & 0.097 (6) & $0.063(4)$ & $0.004(4)$ & $0.037(3)$ & $0.020(4)$ \\
\hline $\mathrm{C} 38$ & $0.033(3)$ & $0.054(4)$ & $0.035(3)$ & -0.008 & $0.011(3)$ & $0.006(3)$ \\
\hline $\mathrm{Br} 3$ & 0.0629 & $0.1340(9)$ & $0.0506(4)$ & $0.0121(5)$ & $0.0425(4)$ & $-0.0018(4)$ \\
\hline $\mathrm{Br} 4$ & $0.0482(4)$ & $0.1339(8)$ & 0.0419 & $0.0164(4)$ & 0.0321 & $0.0144(4)$ \\
\hline $\mathrm{Co} 2$ & $0.0329(4)$ & $0.0487(5)$ & $0.0187(3)$ & $-0.0001(4)$ & 0.0099 & $-0.0015(3)$ \\
\hline $\mathrm{O} 1$ & $0.036(2)$ & $0.066(3)$ & $0.0241(17)$ & $0.003(2)$ & $0.0122(16)$ & $-0.0008(18)$ \\
\hline $\mathrm{O} 4$ & $0.035(2)$ & $0.061(3)$ & 0.0247 (17) & $-0.005(2)$ & $0.0129(16)$ & $-0.0056(18)$ \\
\hline N6 & $0.030(2)$ & $0.047(3)$ & 0.0204 (19) & $-0.006(2)$ & $0.0116(18)$ & $-0.0050(19)$ \\
\hline N5 & $0.031(2)$ & $0.038(3)$ & 0.0220 (19) & $-0.002(2)$ & $0.0120(18)$ & $-0.0006(18)$ \\
\hline N7 & $0.027(2)$ & $0.046(3)$ & 0.0221 (19) & $0.012(2)$ & $0.0103(18)$ & 0.0069 (19) \\
\hline
\end{tabular}


supporting information

$\begin{array}{lllllll}\mathrm{N} 8 & 0.029(2) & 0.054(3) & 0.0173(18) & -0.005(2) & 0.0098(18) & -0.004(2) \\ \mathrm{C} 13 & 0.030(3) & 0.049(4) & 0.029(3) & -0.012(3) & 0.014(2) & -0.010(2) \\ \mathrm{C} 21 & 0.027(3) & 0.053(4) & 0.025(2) & 0.005(3) & 0.014(2) & 0.001(2) \\ \mathrm{C} 14 & 0.029(3) & 0.046(4) & 0.022(2) & 0.013(3) & 0.014(2) & 0.003(2) \\ \mathrm{C} 8 & 0.027(3) & 0.047(4) & 0.029(2) & -0.014(3) & 0.014(2) & -0.011(2) \\ \mathrm{C} 3 & 0.044(3) & 0.051(4) & 0.027(3) & -0.003(3) & 0.017(3) & -0.005(3) \\ \mathrm{C} 19 & 0.032(3) & 0.035(4) & 0.050(3) & 0.001(3) & 0.016(3) & -0.007(3) \\ \mathrm{C} 6 & 0.041(3) & 0.050(4) & 0.024(2) & -0.001(3) & 0.014(2) & 0.007(2) \\ \text { C2 } & 0.028(3) & 0.035(3) & 0.022(2) & -0.005(3) & 0.009(2) & 0.000(2) \\ \text { C9 } & 0.037(3) & 0.047(4) & 0.031(3) & -0.013(3) & 0.019(2) & -0.006(2) \\ \text { C4 } & 0.045(4) & 0.064(5) & 0.038(3) & 0.007(3) & 0.026(3) & -0.003(3) \\ \text { C15 } & 0.034(3) & 0.036(4) & 0.031(3) & 0.019(3) & 0.018(2) & 0.010(2) \\ \text { C26 } & 0.031(3) & 0.061(4) & 0.025(2) & 0.006(3) & 0.014(2) & 0.002(3) \\ \text { C1 } & 0.028(3) & 0.038(4) & 0.025(2) & -0.010(3) & 0.012(2) & -0.006(2) \\ \text { C10 } & 0.036(3) & 0.061(5) & 0.042(3) & -0.013(3) & 0.024(3) & -0.010(3) \\ \text { C17 } & 0.047(4) & 0.031(4) & 0.072(4) & 0.005(3) & 0.039(3) & 0.012(3) \\ \text { C20 } & 0.027(3) & 0.021(3) & 0.038(3) & 0.001(2) & 0.016(2) & -0.003(2) \\ \text { C24 } & 0.037(3) & 0.072(5) & 0.057(4) & -0.003(3) & 0.032(3) & 0.009(3) \\ \text { C5 } & 0.037(3) & 0.055(5) & 0.044(3) & 0.009(3) & 0.020(3) & 0.005(3) \\ \text { C7 } & 0.028(3) & 0.036(3) & 0.026(2) & -0.009(3) & 0.015(2) & -0.004(2) \\ \text { C25 } & 0.032(3) & 0.058(4) & 0.038(3) & -0.017(3) & 0.015(3) & -0.005(3) \\ \text { C16 } & 0.042(3) & 0.031(3) & 0.040(3) & 0.006(3) & 0.023(3) & 0.006(3) \\ \text { C22 } & 0.035(3) & 0.073(5) & 0.029(3) & 0.012(3) & 0.018(3) & 0.007(3) \\ \text { C12 } & 0.034(3) & 0.044(4) & 0.041(3) & 0.009(3) & 0.017(3) & 0.002(3) \\ \text { C23 } & 0.038(3) & 0.068(5) & 0.038(3) & 0.009(3) & 0.026(3) & 0.008(3) \\ \text { C11 } & 0.039(3) & 0.047(4) & 0.051(3) & -0.005(3) & 0.028(3) & -0.005(3) \\ \text { C18 } & 0.032(3) & 0.038(4) & 0.068(4) & 0.004(3) & 0.025(3) & 0.000(3) \\ & & & & & \end{array}$

Geometric parameters $\left(\AA,{ }^{\circ}\right)$

\begin{tabular}{llll}
\hline $\mathrm{Br} 1-\mathrm{C} 49$ & $1.904(5)$ & $\mathrm{Br}-\mathrm{C} 10$ & $1.889(6)$ \\
$\mathrm{Br} 2-\mathrm{C} 36$ & $1.898(5)$ & $\mathrm{Br}-\mathrm{C} 23$ & $1.893(6)$ \\
$\mathrm{Co} 1-\mathrm{O} 2$ & $1.912(4)$ & $\mathrm{C} 2-\mathrm{O} 1$ & $1.930(4)$ \\
$\mathrm{Co} 1-\mathrm{O} 3$ & $1.903(4)$ & $\mathrm{C} 2-\mathrm{O} 4$ & $1.912(4)$ \\
$\mathrm{Co} 1-\mathrm{N} 2$ & $1.975(4)$ & $\mathrm{Co}-\mathrm{N} 6$ & $1.961(4)$ \\
$\mathrm{Co} 1-\mathrm{N} 3$ & $1.967(4)$ & $\mathrm{C} 2-\mathrm{N} 7$ & $1.957(4)$ \\
$\mathrm{O} 2-\mathrm{C} 52$ & $1.328(5)$ & $\mathrm{O} 1-\mathrm{C} 13$ & $1.322(6)$ \\
$\mathrm{O} 3-\mathrm{C} 39$ & $1.325(5)$ & $\mathrm{O} 4-\mathrm{C} 26$ & $1.316(6)$ \\
$\mathrm{N} 2-\mathrm{C} 40$ & $1.339(6)$ & $\mathrm{N} 6-\mathrm{C} 1$ & $1.332(6)$ \\
$\mathrm{N} 2-\mathrm{C} 41$ & $1.399(6)$ & $\mathrm{N} 6-\mathrm{C} 2$ & $1.388(7)$ \\
$\mathrm{N} 1-\mathrm{C} 40$ & $1.358(6)$ & $\mathrm{N} 5-\mathrm{C} 1$ & $1.385(6)$ \\
$\mathrm{N} 1-\mathrm{C} 46$ & $1.390(6)$ & $\mathrm{N} 5-\mathrm{C} 7$ & $1.396(7)$ \\
$\mathrm{N} 1-\mathrm{H} 1 \mathrm{~A}$ & 0.8600 & $\mathrm{~N} 5-\mathrm{H} 5 \mathrm{~A}$ & 0.8600 \\
$\mathrm{~N} 3-\mathrm{C} 27$ & $1.336(6)$ & $\mathrm{N} 7-\mathrm{C} 14$ & $1.330(6)$ \\
$\mathrm{N} 3-\mathrm{C} 28$ & $1.389(7)$ & $\mathrm{N} 7-\mathrm{C} 15$ & $1.388(7)$ \\
$\mathrm{N} 4-\mathrm{C} 27$ & $1.366(6)$ & $\mathrm{N} 8-\mathrm{C} 14$ & $1.372(6)$ \\
$\mathrm{N} 4-\mathrm{C} 33$ & $1.375(7)$ & $\mathrm{N} 8-\mathrm{C} 20$ & $1.384(7)$ \\
$\mathrm{N} 4-\mathrm{H} 4 \mathrm{~A}$ & 0.8600 & $\mathrm{~N} 8-\mathrm{H} 8 \mathrm{~A}$ & 0.8600
\end{tabular}




\begin{tabular}{|c|c|c|c|}
\hline $\mathrm{C} 41-\mathrm{C} 42$ & $1.378(8)$ & $\mathrm{C} 13-\mathrm{C} 12$ & $1.400(8)$ \\
\hline $\mathrm{C} 41-\mathrm{C} 46$ & $1.392(7)$ & $\mathrm{C} 13-\mathrm{C} 8$ & $1.412(8)$ \\
\hline $\mathrm{C} 28-\mathrm{C} 33$ & $1.388(7)$ & $\mathrm{C} 21-\mathrm{C} 22$ & $1.408(7)$ \\
\hline $\mathrm{C} 28-\mathrm{C} 29$ & $1.406(8)$ & $\mathrm{C} 21-\mathrm{C} 26$ & $1.424(8)$ \\
\hline $\mathrm{C} 36-\mathrm{C} 35$ & $1.334(8)$ & $\mathrm{C} 21-\mathrm{C} 14$ & $1.430(8)$ \\
\hline $\mathrm{C} 36-\mathrm{C} 37$ & $1.391(8)$ & $\mathrm{C} 8-\mathrm{C} 9$ & $1.406(7)$ \\
\hline $\mathrm{C} 40-\mathrm{C} 47$ & $1.462(7)$ & $\mathrm{C} 8-\mathrm{C} 1$ & $1.447(8)$ \\
\hline $\mathrm{C} 27-\mathrm{C} 34$ & $1.448(7)$ & $\mathrm{C} 3-\mathrm{C} 4$ & $1.369(8)$ \\
\hline $\mathrm{C} 46-\mathrm{C} 45$ & $1.377(7)$ & $\mathrm{C} 3-\mathrm{C} 2$ & $1.389(7)$ \\
\hline $\mathrm{C} 45-\mathrm{C} 44$ & $1.362(8)$ & $\mathrm{C} 3-\mathrm{H} 3$ & 0.9300 \\
\hline $\mathrm{C} 45-\mathrm{H} 45$ & 0.9300 & $\mathrm{C} 19-\mathrm{C} 18$ & $1.351(9)$ \\
\hline $\mathrm{C} 48-\mathrm{C} 49$ & $1.350(7)$ & $\mathrm{C} 19-\mathrm{C} 20$ & $1.382(8)$ \\
\hline $\mathrm{C} 48-\mathrm{C} 47$ & $1.405(7)$ & $\mathrm{C} 19-\mathrm{H} 19$ & 0.9300 \\
\hline $\mathrm{C} 48-\mathrm{H} 48$ & 0.9300 & $\mathrm{C} 6-\mathrm{C} 5$ & $1.376(8)$ \\
\hline $\mathrm{C} 33-\mathrm{C} 32$ & $1.381(8)$ & $\mathrm{C} 6-\mathrm{C} 7$ & $1.381(7)$ \\
\hline $\mathrm{C} 49-\mathrm{C} 50$ & $1.391(7)$ & $\mathrm{C} 6-\mathrm{H} 6$ & 0.9300 \\
\hline $\mathrm{C} 35-\mathrm{C} 34$ & $1.414(6)$ & $\mathrm{C} 2-\mathrm{C} 7$ & $1.401(6)$ \\
\hline $\mathrm{C} 35-\mathrm{H} 35$ & 0.9300 & $\mathrm{C} 9-\mathrm{C} 10$ & $1.359(8)$ \\
\hline $\mathrm{C} 34-\mathrm{C} 39$ & $1.410(7)$ & $\mathrm{C} 9-\mathrm{H} 9$ & 0.9300 \\
\hline $\mathrm{C} 43-\mathrm{C} 42$ & $1.385(8)$ & $\mathrm{C} 4-\mathrm{C} 5$ & $1.378(8)$ \\
\hline $\mathrm{C} 43-\mathrm{C} 44$ & $1.403(8)$ & $\mathrm{C} 4-\mathrm{H} 4$ & 0.9300 \\
\hline $\mathrm{C} 43-\mathrm{H} 43$ & 0.9300 & $\mathrm{C} 15-\mathrm{C} 16$ & $1.399(7)$ \\
\hline $\mathrm{C} 44-\mathrm{H} 44$ & 0.9300 & $\mathrm{C} 15-\mathrm{C} 20$ & $1.403(7)$ \\
\hline $\mathrm{C} 31-\mathrm{C} 32$ & $1.386(9)$ & $\mathrm{C} 26-\mathrm{C} 25$ & $1.420(8)$ \\
\hline $\mathrm{C} 31-\mathrm{C} 30$ & $1.391(9)$ & $\mathrm{C} 10-\mathrm{C} 11$ & $1.387(8)$ \\
\hline $\mathrm{C} 31-\mathrm{H} 31$ & 0.9300 & $\mathrm{C} 17-\mathrm{C} 16$ & $1.370(8)$ \\
\hline $\mathrm{C} 50-\mathrm{C} 51$ & $1.354(7)$ & $\mathrm{C} 17-\mathrm{C} 18$ & $1.385(9)$ \\
\hline $\mathrm{C} 50-\mathrm{H} 50$ & 0.9300 & $\mathrm{C} 17-\mathrm{H} 17$ & 0.9300 \\
\hline $\mathrm{C} 47-\mathrm{C} 52$ & $1.417(6)$ & $\mathrm{C} 24-\mathrm{C} 25$ & $1.361(8)$ \\
\hline $\mathrm{C} 42-\mathrm{H} 42$ & 0.9300 & $\mathrm{C} 24-\mathrm{C} 23$ & $1.391(9)$ \\
\hline $\mathrm{C} 37-\mathrm{C} 38$ & $1.375(7)$ & $\mathrm{C} 24-\mathrm{H} 24$ & 0.9300 \\
\hline C $37-\mathrm{H} 37$ & 0.9300 & $\mathrm{C} 5-\mathrm{H} 5$ & 0.9300 \\
\hline C51-C52 & $1.383(7)$ & $\mathrm{C} 25-\mathrm{H} 25$ & 0.9300 \\
\hline C51-H51 & 0.9300 & $\mathrm{C} 16-\mathrm{H} 16$ & 0.9300 \\
\hline C $32-\mathrm{H} 32$ & 0.9300 & $\mathrm{C} 22-\mathrm{C} 23$ & $1.351(9)$ \\
\hline $\mathrm{C} 39-\mathrm{C} 38$ & $1.382(8)$ & $\mathrm{C} 22-\mathrm{H} 22$ & 0.9300 \\
\hline $\mathrm{C} 29-\mathrm{C} 30$ & $1.347(8)$ & $\mathrm{C} 12-\mathrm{C} 11$ & $1.364(8)$ \\
\hline $\mathrm{C} 29-\mathrm{H} 29$ & 0.9300 & $\mathrm{C} 12-\mathrm{H} 12$ & 0.9300 \\
\hline $\mathrm{C} 30-\mathrm{H} 30$ & 0.9300 & $\mathrm{C} 11-\mathrm{H} 11$ & 0.9300 \\
\hline C $38-\mathrm{H} 38$ & 0.9300 & $\mathrm{C} 18-\mathrm{H} 18$ & 0.9300 \\
\hline $\mathrm{O} 3-\mathrm{Co} 1-\mathrm{O} 2$ & $107.28(18)$ & $\mathrm{O} 4-\mathrm{Co} 2-\mathrm{O} 1$ & 125.57 (19) \\
\hline $\mathrm{O} 3-\mathrm{Co} 1-\mathrm{N} 3$ & $93.74(17)$ & $\mathrm{O} 4-\mathrm{Co} 2-\mathrm{N} 7$ & 94.49 (17) \\
\hline $\mathrm{O} 2-\mathrm{Co} 1-\mathrm{N} 3$ & $121.01(17)$ & $\mathrm{O} 1-\mathrm{Co} 2-\mathrm{N} 7$ & $108.97(17)$ \\
\hline $\mathrm{O} 3-\mathrm{Co} 1-\mathrm{N} 2$ & $121.83(17)$ & $\mathrm{O} 4-\mathrm{Co} 2-\mathrm{N} 6$ & $113.60(17)$ \\
\hline $\mathrm{O} 2-\mathrm{Co} 1-\mathrm{N} 2$ & $92.47(15)$ & $\mathrm{O} 1-\mathrm{Co} 2-\mathrm{N} 6$ & $93.86(17)$ \\
\hline $\mathrm{N} 3-\mathrm{Co} 1-\mathrm{N} 2$ & $121.93(17)$ & $\mathrm{N} 7-\mathrm{Co} 2-\mathrm{N} 6$ & $123.0(2)$ \\
\hline $\mathrm{C} 52-\mathrm{O} 2-\mathrm{Co} 1$ & $123.7(3)$ & $\mathrm{C} 13-\mathrm{O} 1-\mathrm{Co} 2$ & $127.3(4)$ \\
\hline
\end{tabular}




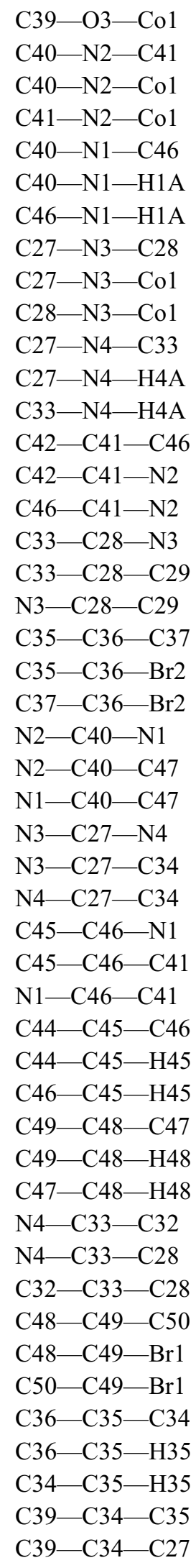

$125.5(3)$

$106.9(4)$

$121.4(3)$

130.4 (3)

$108.6(4)$

125.7

125.7

106.7 (4)

$121.0(4)$

$131.4(3)$

109.1 (4)

125.4

125.4

$121.4(5)$

$130.0(5)$

$108.6(5)$

$109.2(5)$

$119.2(5)$

$131.5(5)$

$121.1(5)$

$121.3(4)$

$117.6(4)$

$110.3(4)$

$125.4(4)$

$124.3(4)$

$109.6(5)$

$126.6(4)$

$123.9(4)$

$132.6(5)$

$121.7(5)$

105.7 (4)

$116.9(5)$

121.5

121.5

$120.8(4)$

119.6

119.6

$132.0(5)$

$105.2(5)$

$122.7(6)$

$120.9(5)$

$121.1(4)$

$118.0(4)$

$121.7(5)$

119.2

119.2

$118.1(5)$

$122.7(4)$
$\mathrm{C} 26-\mathrm{O} 4-\mathrm{Co} 2$

$\mathrm{C} 1-\mathrm{N} 6-\mathrm{C} 2$

$\mathrm{C} 1-\mathrm{N} 6-\mathrm{Co} 2$

$\mathrm{C} 2-\mathrm{N} 6-\mathrm{Co} 2$

C1-N5-C7

$\mathrm{C} 1-\mathrm{N} 5-\mathrm{H} 5 \mathrm{~A}$

C7-N5-H5A

C14-N7-C15

$\mathrm{C} 14-\mathrm{N} 7-\mathrm{Co} 2$

$\mathrm{C} 15-\mathrm{N} 7-\mathrm{Co} 2$

$\mathrm{C} 14-\mathrm{N} 8-\mathrm{C} 20$

C14-N8-H8A

$\mathrm{C} 20-\mathrm{N} 8-\mathrm{H} 8 \mathrm{~A}$

$\mathrm{O} 1-\mathrm{C} 13-\mathrm{C} 12$

$\mathrm{O} 1-\mathrm{C} 13-\mathrm{C} 8$

$\mathrm{C} 12-\mathrm{C} 13-\mathrm{C} 8$

$\mathrm{C} 22-\mathrm{C} 21-\mathrm{C} 26$

$\mathrm{C} 22-\mathrm{C} 21-\mathrm{C} 14$

$\mathrm{C} 26-\mathrm{C} 21-\mathrm{C} 14$

$\mathrm{N} 7-\mathrm{C} 14-\mathrm{N} 8$

$\mathrm{N} 7-\mathrm{C} 14-\mathrm{C} 21$

$\mathrm{N} 8-\mathrm{C} 14-\mathrm{C} 21$

C9- 8 - $\mathrm{C} 13$

$\mathrm{C} 9-\mathrm{C} 8-\mathrm{C} 1$

$\mathrm{C} 13-\mathrm{C} 8-\mathrm{C} 1$

$\mathrm{C} 4-\mathrm{C} 3-\mathrm{C} 2$

$\mathrm{C} 4-\mathrm{C} 3-\mathrm{H} 3$

$\mathrm{C} 2-\mathrm{C} 3-\mathrm{H} 3$

$\mathrm{C} 18-\mathrm{C} 19-\mathrm{C} 20$

C18-C19-H19

C20-C19-H19

C5-C6-C7

$\mathrm{C} 5-\mathrm{C} 6-\mathrm{H} 6$

$\mathrm{C} 7-\mathrm{C} 6-\mathrm{H} 6$

$\mathrm{N} 6-\mathrm{C} 2-\mathrm{C} 3$

$\mathrm{N} 6-\mathrm{C} 2-\mathrm{C} 7$

$\mathrm{C} 3-\mathrm{C} 2-\mathrm{C} 7$

$\mathrm{C} 10-\mathrm{C} 9-\mathrm{C} 8$

$\mathrm{C} 10-\mathrm{C} 9-\mathrm{H} 9$

$\mathrm{C} 8-\mathrm{C} 9-\mathrm{H} 9$

$\mathrm{C} 3-\mathrm{C} 4-\mathrm{C} 5$

$\mathrm{C} 3-\mathrm{C} 4-\mathrm{H} 4$

$\mathrm{C} 5-\mathrm{C} 4-\mathrm{H} 4$

N7-C15-C16

$\mathrm{N} 7-\mathrm{C} 15-\mathrm{C} 20$

$\mathrm{C} 16-\mathrm{C} 15-\mathrm{C} 20$

O4-C26-C25

O4-C26-C21
$126.5(4)$

$107.9(4)$

124.0 (4)

127.7 (3)

109.5 (4)

125.3

125.3

107.2 (4)

123.4 (4)

$128.2(3)$

109.3 (4)

125.4

125.4

117.4 (5)

125.4 (5)

$117.2(5)$

$118.3(5)$

119.4 (5)

$122.3(5)$

$109.7(5)$

$126.3(5)$

$124.0(4)$

$119.0(5)$

$118.7(5)$

$122.3(5)$

118.1 (5)

121.0

121.0

115.6 (6)

122.2

122.2

115.8 (5)

122.1

122.1

$130.6(4)$

109.7 (4)

119.7 (5)

121.5 (6)

119.2

119.2

121.0 (6)

119.5

119.5

$132.2(5)$

109.4 (5)

118.4 (5)

$117.2(5)$

$125.6(5)$ 


\begin{tabular}{|c|c|}
\hline $\mathrm{C} 35-\mathrm{C} 34-\mathrm{C} 27$ & $119.1(4)$ \\
\hline $\mathrm{C} 42-\mathrm{C} 43-\mathrm{C} 44$ & $120.6(6)$ \\
\hline $\mathrm{C} 42-\mathrm{C} 43-\mathrm{H} 43$ & 119.7 \\
\hline $\mathrm{C} 44-\mathrm{C} 43-\mathrm{H} 43$ & 119.7 \\
\hline $\mathrm{C} 45-\mathrm{C} 44-\mathrm{C} 43$ & $122.3(5)$ \\
\hline $\mathrm{C} 45-\mathrm{C} 44-\mathrm{H} 44$ & 118.9 \\
\hline $\mathrm{C} 43-\mathrm{C} 44-\mathrm{H} 44$ & 118.9 \\
\hline $\mathrm{C} 32-\mathrm{C} 31-\mathrm{C} 30$ & $121.9(6)$ \\
\hline $\mathrm{C} 32-\mathrm{C} 31-\mathrm{H} 31$ & 119.1 \\
\hline $\mathrm{C} 30-\mathrm{C} 31-\mathrm{H} 31$ & 119.1 \\
\hline $\mathrm{C} 51-\mathrm{C} 50-\mathrm{C} 49$ & $119.2(5)$ \\
\hline $\mathrm{C} 51-\mathrm{C} 50-\mathrm{H} 50$ & 120.4 \\
\hline $\mathrm{C} 49-\mathrm{C} 50-\mathrm{H} 50$ & 120.4 \\
\hline $\mathrm{C} 48-\mathrm{C} 47-\mathrm{C} 52$ & $118.3(4)$ \\
\hline $\mathrm{C} 48-\mathrm{C} 47-\mathrm{C} 40$ & $119.1(4)$ \\
\hline $\mathrm{C} 52-\mathrm{C} 47-\mathrm{C} 40$ & $122.5(4)$ \\
\hline $\mathrm{C} 41-\mathrm{C} 42-\mathrm{C} 43$ & $117.1(5)$ \\
\hline $\mathrm{C} 41-\mathrm{C} 42-\mathrm{H} 42$ & 121.5 \\
\hline $\mathrm{C} 43-\mathrm{C} 42-\mathrm{H} 42$ & 121.5 \\
\hline $\mathrm{C} 38-\mathrm{C} 37-\mathrm{C} 36$ & $118.0(6)$ \\
\hline $\mathrm{C} 38-\mathrm{C} 37-\mathrm{H} 37$ & 121.0 \\
\hline $\mathrm{C} 36-\mathrm{C} 37-\mathrm{H} 37$ & 121.0 \\
\hline $\mathrm{C} 50-\mathrm{C} 51-\mathrm{C} 52$ & $122.2(5)$ \\
\hline $\mathrm{C} 50-\mathrm{C} 51-\mathrm{H} 51$ & 118.9 \\
\hline $\mathrm{C} 52-\mathrm{C} 51-\mathrm{H} 51$ & 118.9 \\
\hline $\mathrm{C} 33-\mathrm{C} 32-\mathrm{C} 31$ & $116.2(6)$ \\
\hline $\mathrm{C} 33-\mathrm{C} 32-\mathrm{H} 32$ & 121.9 \\
\hline $\mathrm{C} 31-\mathrm{C} 32-\mathrm{H} 32$ & 121.9 \\
\hline $\mathrm{O} 3-\mathrm{C} 39-\mathrm{C} 38$ & $117.6(5)$ \\
\hline $\mathrm{O} 3-\mathrm{C} 39-\mathrm{C} 34$ & $124.3(5)$ \\
\hline $\mathrm{C} 38-\mathrm{C} 39-\mathrm{C} 34$ & $118.1(4)$ \\
\hline $\mathrm{O} 2-\mathrm{C} 52-\mathrm{C} 51$ & $117.5(4)$ \\
\hline $\mathrm{O} 2-\mathrm{C} 52-\mathrm{C} 47$ & $124.0(5)$ \\
\hline $\mathrm{C} 51-\mathrm{C} 52-\mathrm{C} 47$ & $118.5(4)$ \\
\hline $\mathrm{C} 30-\mathrm{C} 29-\mathrm{C} 28$ & $118.7(6)$ \\
\hline $\mathrm{C} 30-\mathrm{C} 29-\mathrm{H} 29$ & 120.7 \\
\hline $\mathrm{C} 28-\mathrm{C} 29-\mathrm{H} 29$ & 120.7 \\
\hline $\mathrm{C} 29-\mathrm{C} 30-\mathrm{C} 31$ & $121.2(6)$ \\
\hline $\mathrm{C} 29-\mathrm{C} 30-\mathrm{H} 30$ & 119.4 \\
\hline $\mathrm{C} 31-\mathrm{C} 30-\mathrm{H} 30$ & 119.4 \\
\hline $\mathrm{C} 37-\mathrm{C} 38-\mathrm{C} 39$ & $123.0(5)$ \\
\hline $\mathrm{C} 37-\mathrm{C} 38-\mathrm{H} 38$ & 118.5 \\
\hline
\end{tabular}

\begin{tabular}{|c|c|}
\hline $\mathrm{C} 25-\mathrm{C} 26-\mathrm{C} 21$ & $117.2(5)$ \\
\hline $\mathrm{N} 6-\mathrm{C} 1-\mathrm{N} 5$ & $108.8(5)$ \\
\hline $\mathrm{N} 6-\mathrm{C} 1-\mathrm{C} 8$ & $126.7(5)$ \\
\hline $\mathrm{N} 5-\mathrm{C} 1-\mathrm{C} 8$ & $124.5(4)$ \\
\hline $\mathrm{C} 9-\mathrm{C} 10-\mathrm{C} 11$ & $120.1(5)$ \\
\hline $\mathrm{C} 9-\mathrm{C} 10-\mathrm{Br} 3$ & $119.9(5)$ \\
\hline $\mathrm{C} 11-\mathrm{C} 10-\mathrm{Br} 3$ & $119.8(5)$ \\
\hline $\mathrm{C} 16-\mathrm{C} 17-\mathrm{C} 18$ & $119.6(6)$ \\
\hline $\mathrm{C} 16-\mathrm{C} 17-\mathrm{H} 17$ & 120.2 \\
\hline $\mathrm{C} 18-\mathrm{C} 17-\mathrm{H} 17$ & 120.2 \\
\hline $\mathrm{C} 19-\mathrm{C} 20-\mathrm{N} 8$ & $132.4(5)$ \\
\hline $\mathrm{C} 19-\mathrm{C} 20-\mathrm{C} 15$ & $123.1(5)$ \\
\hline $\mathrm{N} 8-\mathrm{C} 20-\mathrm{C} 15$ & $104.5(4)$ \\
\hline $\mathrm{C} 25-\mathrm{C} 24-\mathrm{C} 23$ & $119.4(6)$ \\
\hline $\mathrm{C} 25-\mathrm{C} 24-\mathrm{H} 24$ & 120.3 \\
\hline $\mathrm{C} 23-\mathrm{C} 24-\mathrm{H} 24$ & 120.3 \\
\hline $\mathrm{C} 6-\mathrm{C} 5-\mathrm{C} 4$ & $123.0(6)$ \\
\hline $\mathrm{C} 6-\mathrm{C} 5-\mathrm{H} 5$ & 118.5 \\
\hline $\mathrm{C} 4-\mathrm{C} 5-\mathrm{H} 5$ & 118.5 \\
\hline $\mathrm{C} 6-\mathrm{C} 7-\mathrm{N} 5$ & $133.4(4)$ \\
\hline $\mathrm{C} 6-\mathrm{C} 7-\mathrm{C} 2$ & $122.5(5)$ \\
\hline $\mathrm{N} 5-\mathrm{C} 7-\mathrm{C} 2$ & $104.1(4)$ \\
\hline $\mathrm{C} 24-\mathrm{C} 25-\mathrm{C} 26$ & $122.5(6)$ \\
\hline $\mathrm{C} 24-\mathrm{C} 25-\mathrm{H} 25$ & 118.7 \\
\hline $\mathrm{C} 26-\mathrm{C} 25-\mathrm{H} 25$ & 118.7 \\
\hline $\mathrm{C} 17-\mathrm{C} 16-\mathrm{C} 15$ & $119.0(5)$ \\
\hline $\mathrm{C} 17-\mathrm{C} 16-\mathrm{H} 16$ & 120.5 \\
\hline $\mathrm{C} 15-\mathrm{C} 16-\mathrm{H} 16$ & 120.5 \\
\hline $\mathrm{C} 23-\mathrm{C} 22-\mathrm{C} 21$ & $122.3(6)$ \\
\hline $\mathrm{C} 23-\mathrm{C} 22-\mathrm{H} 22$ & 118.8 \\
\hline $\mathrm{C} 21-\mathrm{C} 22-\mathrm{H} 22$ & 118.8 \\
\hline $\mathrm{C} 11-\mathrm{C} 12-\mathrm{C} 13$ & $123.0(5)$ \\
\hline $\mathrm{C} 11-\mathrm{C} 12-\mathrm{H} 12$ & 118.5 \\
\hline $\mathrm{C} 13-\mathrm{C} 12-\mathrm{H} 12$ & 118.5 \\
\hline $\mathrm{C} 22-\mathrm{C} 23-\mathrm{C} 24$ & $120.3(6)$ \\
\hline $\mathrm{C} 22-\mathrm{C} 23-\mathrm{Br} 4$ & $120.3(5)$ \\
\hline $\mathrm{C} 24-\mathrm{C} 23-\mathrm{Br} 4$ & $119.4(5)$ \\
\hline $\mathrm{C} 12-\mathrm{C} 11-\mathrm{C} 10$ & $119.1(6)$ \\
\hline $\mathrm{C} 12-\mathrm{C} 11-\mathrm{H} 11$ & 120.4 \\
\hline $\mathrm{C} 10-\mathrm{C} 11-\mathrm{H} 11$ & 120.4 \\
\hline $\mathrm{C} 19-\mathrm{C} 18-\mathrm{C} 17$ & $124.3(6)$ \\
\hline $\mathrm{C} 19-\mathrm{C} 18-\mathrm{H} 18$ & 117.8 \\
\hline $\mathrm{C} 17-\mathrm{C} 18-\mathrm{H} 18$ & 117.8 \\
\hline
\end{tabular}

Hydrogen-bond geometry $\left(A,{ }^{\circ}\right)$

\begin{tabular}{lllll}
\hline$D-\mathrm{H} \cdots A$ & $D-\mathrm{H}$ & $\mathrm{H} \cdots A$ & $D \cdots A$ & $D-\mathrm{H}^{\cdots} A A$ \\
\hline $\mathrm{N} 1-\mathrm{H} 1 A \cdots \mathrm{O} 1^{\mathrm{i}}$ & 0.86 & 2.19 & $2.895(5)$ & 139
\end{tabular}


supporting information

$\begin{array}{lllll}\mathrm{N} 4-\mathrm{H} 4 A \cdots \mathrm{O} 2^{\mathrm{ii}} & 0.86 & 2.09 & 2.849(5) & 147 \\ \mathrm{~N} 5-\mathrm{H} 5 A \cdots \mathrm{O} 4^{i i i} & 0.86 & 2.41 & 3.066(5) & 133 \\ \mathrm{~N} 8-\mathrm{H} 8 A \cdots \mathrm{O} 3^{\text {iv }} & 0.86 & 2.17 & 2.808(5) & 131 \\ \mathrm{C} 4-\mathrm{H} 4 \cdots \mathrm{Br} 4^{\mathrm{i}} & 0.93 & 2.88 & 3.703(6) & 149 \\ \mathrm{C} 6-\mathrm{H} 6 \cdots \mathrm{Br}^{\mathrm{v}} & 0.93 & 2.91 & 3.685(6) & 142 \\ \mathrm{C} 35-\mathrm{H} 35 \cdots \mathrm{O} 2^{\mathrm{ii}} & 0.93 & 2.54 & 3.394(7) & 153\end{array}$

Symmetry codes: (i) $-x+1, y-1 / 2,-z+3 / 2$; (ii) $-x+2,-y+1,-z+2$; (iii) $-x+1,-y+2,-z+2$; (iv) $-x+1, y+1 / 2,-z+3 / 2$; (v) $x,-y+3 / 2, z+1 / 2$. 